Gut, 1986, 27, 255-259

\title{
Is there a biochemical basis for lower oesophageal sphincter (LOS) resting pressure?
}

\author{
G ZANINOTTO, L DALLA LIBERA, S MERIGLIANO, AND E ANCONA \\ From the Department of Surgery, University of Padova, and the CNR Unit for Muscle Biology and \\ Physiopathology, Institute of General Pathology, University of Padova, Padova, Italy.
}

SUmmary Myosin plays a fundamental role in the contraction of muscle cells. Some structural differences of this protein are related to the different physical properties of muscle - that is, speed of shortening. The typical myosin protein has a molecular weight of 460000 daltons and is made up of six subunits: two heavy chains (200 000 daltons each) and two pairs of light chains (LC) weighing 15 000-25 000 daltons each (LC1 and LC2). Myosin was extracted from six specimens of human oesophagus in order to find out whether any biochemical differences exist between the oesophageal body and the lower oesophageal sphincter, to account for their different properties. Myosin was examined by two dimensional gel electrophoresis. Peptide mapping of myosin heavy chains was obtained by carrying out enzymatic digestion during the electrophoretic run. A quantitative difference between LC1 of the oesophageal body and the lower oesophageal sphincter was found in the circular fibres, thereby suggesting that two populations of muscle fibres exist in the inner coat of the oesophagus. The presence of more than one type of myosin strongly implies functional differentiation of the two specialised zones.

The lower oesophageal sphincter is widely accepted as a distinct entity differing from the oesophageal body in its physiological, ${ }^{1}$ and pharmacological, ${ }^{2}$ and metabolic ${ }^{3}$ behaviour. The lower oesophageal sphincter is contracted at rest and relaxes on swallowing and vagal stimulation, whereas the oesophageal body relaxes at rest and swallowing causes peristaltic contraction.

The origin of the lower oesophageal sphincter resting pressure is not fully understood and may be because of hormonal or neural influences, myogenic characteristics, or mechanical factors.

One of the mechanical properties of muscle, speed of contraction, is generally proportional to ATPase activity of the muscle myosin, ${ }^{4}$ and differences in ATPase activity reflect differences in myosin structure. ${ }^{5}$ Myosin is the principal element of muscle contractility. It is a structural protein laid down in thick myofilaments, and optimises interaction with the other major contractile protein, actin. Myosin is also an enzyme facilitating conversion of the chemical energy of ATP into motion or force.

It is generally accepted that myosins from skeletal, smooth muscle and non-muscle systems are com-

Address for correspondence: Dr G Zaninotto, Clinica Chirurgica, Policlinico Universitario, Via Giustimiani 2, 35100 Padova, Italy.

Received for publication 5 July 1985 posed of two heavy chains of about 200000 daltons (HC) and two to three light chains (LC), depending on the type of myosin. For example, skeletal muscle myosin consists of three light chains of molecular weight 25000 (LC1), 18000 (LC2), and 16000 daltons (LC3), while the smooth and non-muscle myosins contain two light chains of molecular weight of 20000 (LC1) and 15 000-17 000 daltons (LC2).

In smooth muscle the 20 kilodalton light chain plays a regulating role and must be phosphorylated so that the myosin is activated by actin. ${ }^{6}$

Different types of myosin are identified in the same tissue (for instance, heart) and their differences are related to muscle properties - that is, speed and duration of contraction, both in normal and in pathological states.

The aim of this work was to verify if any differences in myosin exist between oesophageal body muscle and lower oesophageal sphincter, and to explain their different myogenic properties.

\section{Methods}

MATERIAL

Specimens of oesophageal body and lower oesophageal sphincter were taken from patients with cervical or upper thoracic oesophageal carcinoma who had undergone total oesophagectomy. Two 


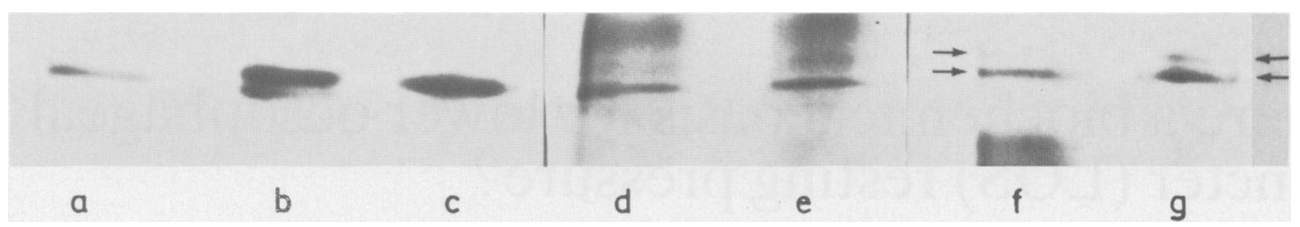

Fig. 1 One dimensional gel electrophoresis of myosin heavy chains from different sources. (a) rat fast-contracting skeletal muscle; (b) $(a)+(c) ;(c)$ rat slow-contracting skeletal muscle; (d) and (e) human platelets; ( $f)$ oesophageal body;

$(g)$ lower oesophageal sphincter: arrows in $(f)$ and $(g)$ indicate oesophageal body and lower oesophageal sphincter myosin heavy chains.

pieces of muscle layer one centimetre in length were dissected immediately over the gastric junction and the other $5-6 \mathrm{~cm}$ proximally, placed in ice cold solution with protease inhibitors, and processed for myosin extraction within 30-60 minutes. In two experiments the surgical material was frozen and stored at $-80^{\circ} \mathrm{C}$. The longitudinal and circular muscle coats of oesophageal body and lower oesophageal sphincter were carefully prepared and separately examined in the two cases.

\section{MYOSIN PREPARATION}

Myosin was isolated from oesophageal body and lower oesophageal sphincter after the procedure described by Fredericksen. ${ }^{7}$ In some experiments myosin was precipitated at the end of the preparation with $50 \%$ ammonium sulphate instead of $25 \mathrm{mmol} \mathrm{Mg}^{2+}$

\section{GEL ELECTROPHORESIS}

Two dimensional gel electrophoresis was done according to O'Farrell ${ }^{8}$ as specifically detailed by Carraro et al. ${ }^{9}$ The frozen surgical material was homogenised in buffer just before the isoelectric point; two-dimensional electrophoresis was done as described above.

\section{PEPTIDE MAPPING OF MYOSIN HEAVY CHAIN}

Peptide mapping of electrophoretically purified myosin heavy chain was carried out according to Cleveland et al ${ }^{10}$ and Rushbrook and Stracher, ${ }^{11}$ as described by Carraro et al. ${ }^{12}$

\section{ANALYSIS OF MYOSIN HEAVY CHAIN}

Intact myosin heavy chains were analysed by gel electrophoresis, according to Carraro and Catani. ${ }^{13}$ All slabs were stained either with Coomassie blue or using the more sensitive silver technique. ${ }^{14}$

\section{Results}

Myosin from most sources has been shown to consist of a heavy chain of about 200000 daltons on sodium-dodecyl-sulphate-polyacrylamide gels, but a remarkable difference was observed with oesophageal body and lower oesophageal sphincter myosins in that their heavy chain consisted of a closely spaced doublet (Fig. 1, rows $f$ and $g$ ). This doublet was seen consistently in all our oesophageal body and lower oesophageal sphincter preparations. In contrast, skeletal muscle myosin yielded only a

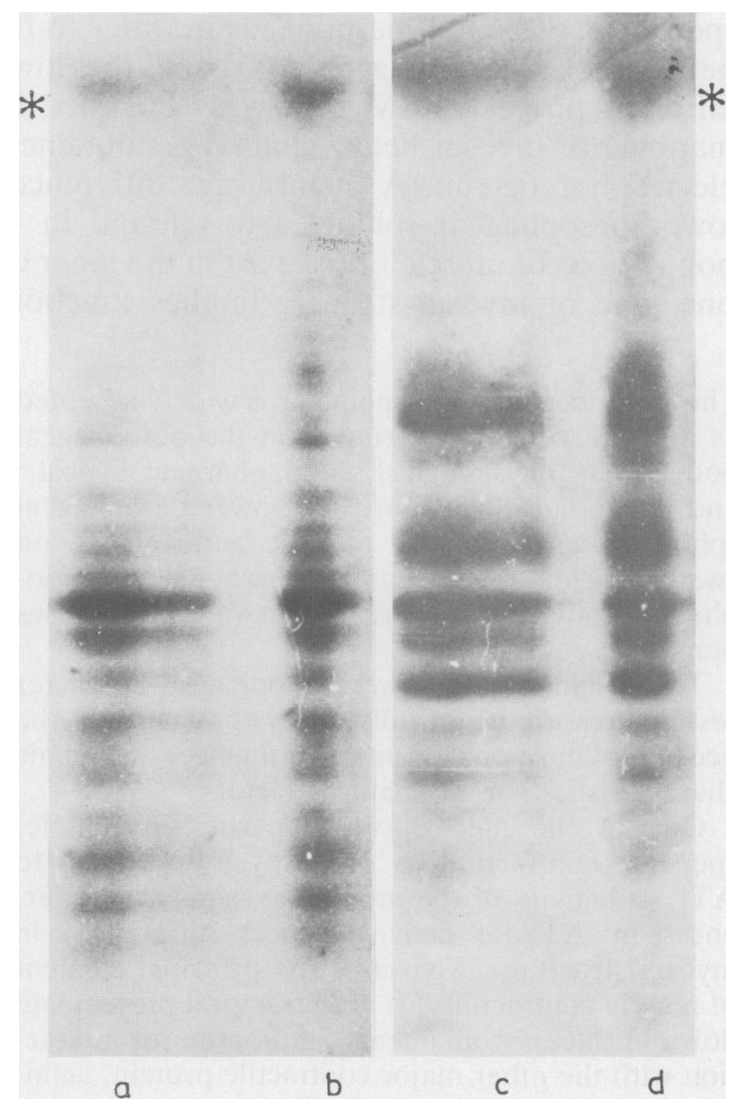

Fig. 2 One-dimensional peptide mapping of electrophoretically purified myosin heavy chains of human gastrointestinal muscle with $S$ aureus $V 8$ protease. (a) and (c) oesophageal body; (b) and (d) lower oesophageal sphincter. Patterns in $(a)$ and $(b)$ are stained with Coomassie brilliant blue; patterns in (c) and (d) are stained with the silver technique. Stars indicate position of $S$ aureus $\checkmark 8$ protease. 
single heavy chain (Fig. 1, rows a,b,c). Myosin from human platelets also showed a single band pattern. (Fig. 1, rows d,e).

The similarity of oesophageal body and lower oesophageal sphincter myosin heavy chains was also confirmed on peptide mapping. The patterns shown in Figure 2 were obtained after digestion of myosin heavy chain with $S$ aureus $V 8$ protease, according to Cleveland et $a l,{ }^{11}$ as described previously. The peptide maps of oesophageal body and lower oesophageal sphincter myosin heavy chains appeared to be identical (Fig. 2, rows a,b) and this similarity was observed with the more sensitive silver stain technique (Fig. 2 rows c,d).

The patterns of oesophageal body and lower oesophageal sphincter myosin light chains at twodimensional gel electrophoresis are shown in Figure 3: in these conditions the myosin light chains were well separated and easily analysed in all the specimens investigated so far. Lower oesophageal sphincter myosin was characterised by two spots I.C1 and LC2 in the light chain region (Fig. 3a) (another contractile protein, can be seen in the slab gel, tropomyosin). Analysis of oesophageal body myosin revealed that LC1 consisted of two polypeptides, characterised by different molecular weight and isoelectric point (Fig. 3b). To show that the irregularly shaped spot in the LC1 region is the result of the overlapping of two separated spots, we show an underexposed picture in which two spots are clearly detectable (Fig. 3d). By contrast LC2 migrates in two dimensional gel as a homogeneous spot. This difference in the number of LC1 light chains may be caused by artefacts during myosin preparation and/or the effect of protease on the myosin molecule. The first possibility was ruled out because similar results were obtained over several experiments. The effect of protease is less likely, as myosin was always prepared in the presence of protease inhibitors such as phenyl-methyl-sulfonylfluoride. In order to rule out the latter possibility, we carried out two-dimensional electrophoresis of muscle samples taken from the patient during the surgical procedure, and then rapidly frozen in liquid nitrogen.

A small piece of frozen muscle sample was homogenised and solubilised in the appropriate buffer before two-dimensional electrophoresis (Methods). In order to test the variable distribution of myosin molecules, not only between lower

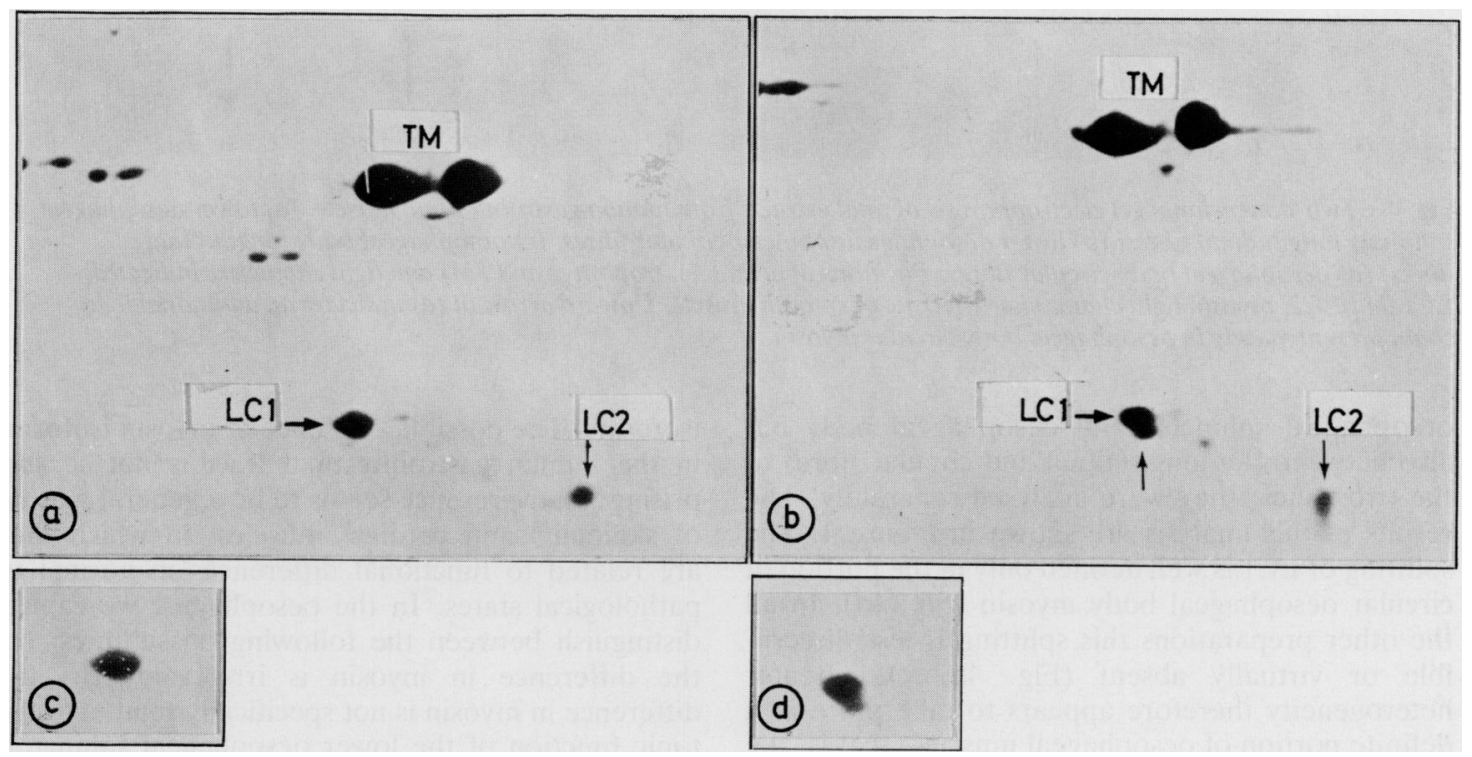

Fig. 3 Two-dimensional gel electrophoresis of myosin light chains from human gastrointestinal muscle. Myosins were purified separately from muscle of adult man: samples were analysed singly $50 \mu \mathrm{g}$ each). Only light chain region of gels is shown. (a) lower oesophageal sphincter myosin; (b) oesophageal body myosin; (c) underexposed and magnified picture of $L C 1$ region of lower oesophageal sphincter. (d): underexposed and magnified picture of $L C 1$ region of oesophageal body. Positions of tropomyosin (TM) and light chains (LC) are indicated. LC1 and LC2: Myosin light chains characteristic of smooth muscles. Upward arrow in (b) indicates an additional light chain present only in oesophageal body myosin, clearly evident in $(d)$. Photos are presented with basic end of isoelectric focusing dimension to the left. 


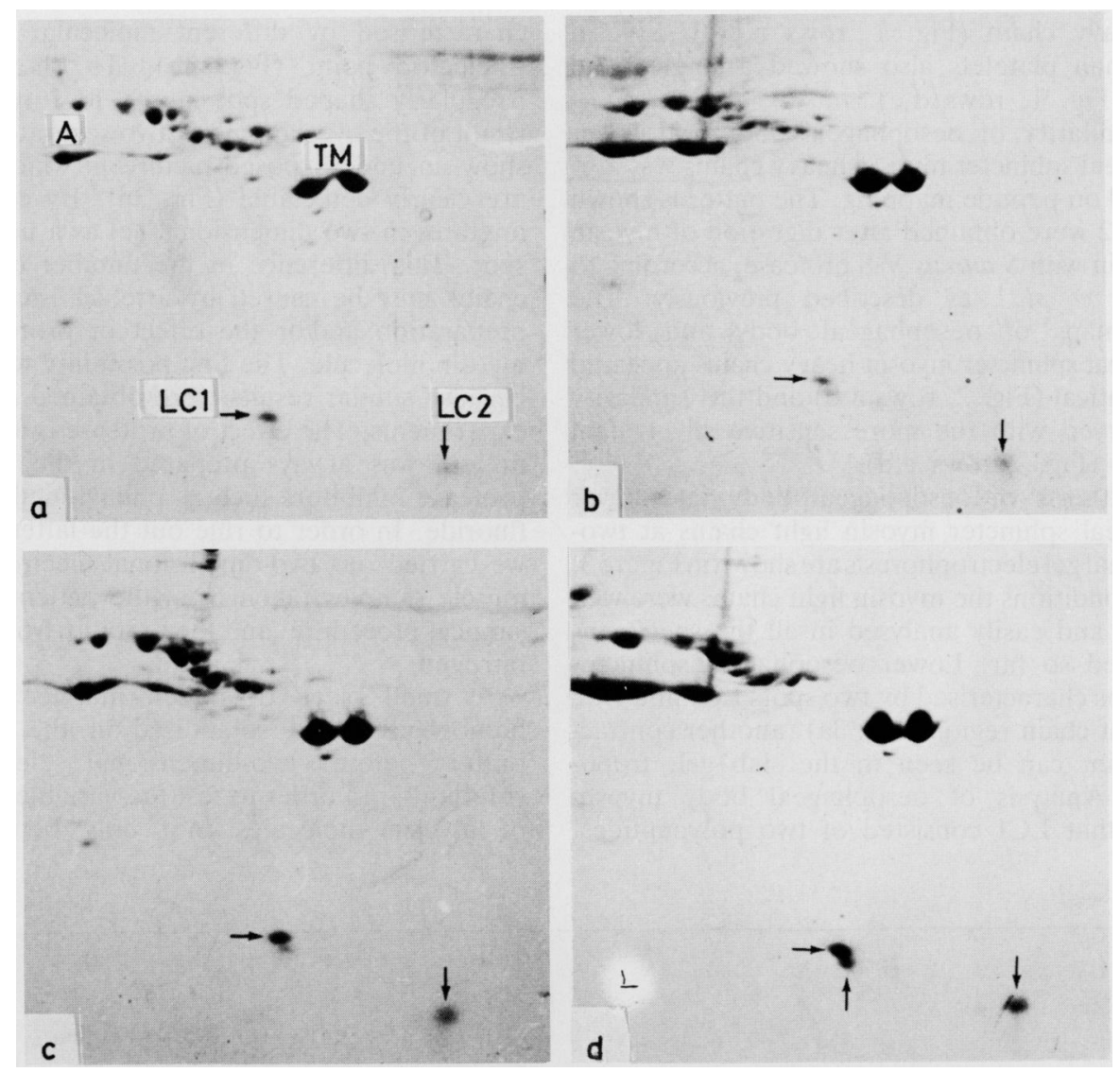

Fig. 4 Two-dimensional gel electrophoresis of total extracts from human gastrointestinal muscle. (a) lower oesophageal sphincter longitudinal fibres; (b) lower oesophageal sphincter circular fibres; (c) oesophageal body longitudinal fibres; (d) oesophageal body circular fibres. Positions of actin (A), tropomyosin (TM) and light chains are indicated. LC1 and LC2: myosin light chains characteristic of smooth muscle. Upward arrow in (d) indicates an additional light chain present mainly in oesophageal body circular myosin.

oesophageal sphincter and oesophageal body but also between the longitudinal and circular fibres of the two zones, they were analysed separately. The results of this analysis are shown in Figure 4. The splitting of LC1 is well defined only in the portion of circular oesophageal body myosin (Fig. 4d). In all the other preparations this splitting is less discernible or virtually absent (Fig. 4a,b,c). Greater heterogeneity therefore appears to take place in a definite portion of oesophageal muscle - that is, the circular fibres.

\section{Discussion}

The data obtained on the subunit composition of oesophageal body and lower oesophageal sphincter myosin may be explained by the presence of myosin isoforms. The possible existence of myosin isoforms in the human gastrointestinal tract is not so surprising: their presence seems to be a general feature of skeletal ${ }^{15}$ and cardiac ${ }^{9}$ muscles, in which they are related to functional differences in normal or pathological states. In the oesophagus, we cannot distinguish between the following possibilities: (a) the difference in myosin is irrelevant; (b) the difference in myosin is not specifically related to the tonic function of the lower oesophageal sphincter; (c) the difference in myosin is secondary to the tonic function; (d) it is the cause of the tonic function. In any case, the presence of more than one type of myosin suggests functional differentiation.

At present the most likely hypothesis for the origin of the basal lower oesophageal sphincter pressure lies in its myogenic properties. This has 
been supported by the finding of differences in the metabolic activities of oesophageal body and lower oesophageal sphincter. Lower oesophageal sphincter has greater dependence on $\mathrm{O}_{2}$ than oesophageal body and has an almost complete aerobic metabolism. ${ }^{3}$ Christensen recently reported that lower oesophageal sphincter has a greater ratio of mitochondria profile area per cell profile area ${ }^{16}$ Furthermore, very recently Fatigati and Murphy have shown that there is a difference in actin isoforms between the lower oesophageal sphincter and the oesophageal body muscle. ${ }^{17}$ Their results suggest that specific actin variants are associated with functional diversity among smooth muscles (normally relaxed muscles contain gamma actin, whereas normally contracted ones contain greater amounts of alpha actin). This too is a quantitative difference, as is our finding concerning myosin LC1 heterogeneity. It is well known that phosphorylation of the LC1 light chain of myosin from smooth muscle is considered to play a role in regulating actinactivated ATPase activity ${ }^{6}$ and self-assembly of the myosins. $^{18}$

Differences in ATPase activity may occur, as the structural differences of smooth muscle myosin isoforms lie in the content of LC1. Myosin isoforms are spatially separated in the circular layer of oesophageal body only. As far as we know, the difference between lower oesophageal sphincter and oesophageal body myosins is due to two different populations of muscle fibres in the inner coat of the oesophagus.

This paper was presented in part at the 2nd Congress of the European Society of Gastrointestinal Motility, Oxford 1984.

\section{References}

1 Castell DO. Lower esophageal sphincter: physiologic and clinical aspects. Ann Intern Med 1975; 83: 390-401.

2 Christensen J. Pharmacology of the oesophageal motor function. Ann Rev Pharmacol 1975; 15: 243-58.

3 Weisbrodt NW, Tague LL. Relationship between contractile and metabolic activities in esophageal muscle. In: Christensen J, ed. Gastrointestinal motility. New York: Raven Press, 1980: 51-7.

4 Barany M. ATPase activity of myosin correlated with speed of muscle shortening. J Gen Physiol 1967; 50: 197-218.
5 Mannherz HG, Goody RS. Proteins of contractile systems. Ann Rev Biochem 1976; 45: 427-65.

6 Adelstein RS, Eisenberg E. Regulation and kinetics of the actin-myosin-ATP interaction. Ann Rev Biochem 1980; 49: 921-56.

7 Frederiksen DW, Rees DD. Preparation of smooth muscle myosin. In: Frederiksen DW, Cunningham LW, eds. Methods in enzymology. Vol. 85. New York: Academic Press, 1982: 292-321.

8 O'Farrell PH. High resolution two dimensional electrophoresis of proteins. J Biol Chem 1975; 250: 4007-21.

9 Carraro U, Dalla Libera L, Catani C. Myosin light chains of avian and mammalian slow muscle: evidence of intraspecific polymorphism. J Muscle Res Cell Motility 1981; 2: 335-42.

10 Cleveland DW, Fischer SG, Kirschner MW, Laemmli UK. Peptide mapping by limited proteolysis in sodium dodecyl sulphate by gel electrophoresis. $J$ Biol Chem 1977; 252: 1102-6.

11 Rushbrook JI, Stracher H. Comparison of adult, embrionic and dystrophic myosin heavy chains from chicken muscle by sodium dodecyl sulphate/polyacrilamide gel electrophoresis and peptide mapping. Proc Natl Acad Sci USA 1979; 76: 4331-4.

12 Carraro U, Catani C, Dalla Libera L. Myosin light and heavy chain in rat gastrocnemius and diaphragm muscle after chronic denervation or reinnervation. Exp Neurol 1981; 72: 401-2.

13 Carraro U, Catani C. A sensitive SDS-PAGE method separating myosin heavy chain isoforms of rat skeletal muscle reveals the heterogenic nature of the embrionic myosin. Biochem Biophys Res Commun 1983; 116: 793-803.

14 Merrill CR, Goldman DX, Sedman SA, Ebert MH. Ultrastructure stain for proteins in polyacrylamide gels shows regional variations in cerebrospinal fluid proteins. Science 1981; 211: 1437-8.

15 Sartore S, Gorza L, Pierobon Bormioli S, Dalla Libera L, Schiaffino S. Myosin types and fiber types in cardiac muscle in ventricular myocardium. J Cell Biol 1981; 88: 226-33.

16 Christensen J. Anatomic specialization in nerve and muscle that defines the lower esophageal sphincter in opossum. In: Demeester TD, Skinner DB, eds. Esophageal disorders pathophysiology and therapy. New York: Raven Press, 1985

17 Fatigati V, Murphy RA. Actin and tropomyosin variants in smooth muscle. Dependence on tissue type. J Biol Chem 1984; 259: 14383-4.

18 Scholey JM, Taylor KA, Kendrick-Jones J. Regulation of non-muscle myosin assembly by calmodulindependent light chain kinase. Nature 1980; 287: 23.3-5. 